\title{
openheart Feasibility and outcomes of ajmaline provocation testing for Brugada syndrome in children in a specialist paediatric inherited cardiovascular diseases centre
}

\author{
Merlin Ranald McMillan, ${ }^{1}$ Thomas George Day, ${ }^{1}$ Margarita Bartsota, ${ }^{1}$ \\ Sarah Mead-Regan, ${ }^{1}$ Rory Bryant, ${ }^{1}$ Jasveer Mangat, ${ }^{1}$ Dominic Abrams, ${ }^{2}$ \\ Martin Lowe, ${ }^{1}$ Juan Pablo Kaski ${ }^{1,3}$
}

To cite: McMillan MR, Day TG, Bartsota M, et al. Feasibility and outcomes of ajmaline provocation testing for Brugada syndrome in children in a specialist paediatric inherited cardiovascular diseases centre. Open Heart 2014;1: e000023. doi:10.1136/ openhrt-2013-000023

- Additional material is published online only. To view please visit the journal online (http://dx.doi.org/10. 1136/openhrt-2013-000023)

Received 16 December 2013 Revised 8 January 2014 Accepted 17 January 2014

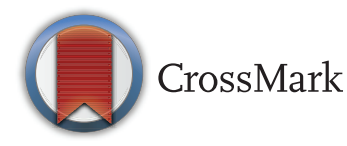

For numbered affiliations see end of article.

Correspondence to Dr Juan Pablo Kaski, Inherited Cardiovascular Diseases Unit, Department of Cardiology, Great Ormond Street Hospital NHS

Foundation Trust, Great Ormond Street, London WC1N 3JH, UK;

j.kaski@ucl.ac.uk

\section{ABSTRACT}

Objectives: Brugada syndrome (BrS) is an inherited arrhythmia syndrome that causes sudden cardiac death in the young. The class la antiarrhythmic ajmaline can be used to provoke the diagnostic ECG pattern. Its use has been established in adults, but little data exist on the ajmaline provocation test in children. This study aims to determine the safety and feasibility of ajmaline provocation testing in a large paediatric cohort in a specialist paediatric inherited cardiac diseases centre.

Methods: 98 consecutive ajmaline tests were performed in 95 children between September 2004 and July 2012 for family history of $\mathrm{BrS}(\mathrm{n}=46(48 \%))$; family history of unexplained sudden cardiac death ( $\mathrm{n}=39(41 \%)$; symptoms with suspicious ECG abnormalities $(n=9(10 \%))$. Three patients were retested with age, due to the possibility of age-related penetrance. ECG parameters were measured at baseline and during maximal ajmaline effect.

Results: The mean patient age was 12.55 years, $43 \%$ were female. Nineteen patients $(20 \%)$ had a positive ajmaline test. There were no arrhythmias or adverse events during testing. Ajmaline provoked significant prolongation of the PR, QRS and QTc in all patients. Mean follow-up was 3.62 years with no adverse outcomes reported in any patients with $\mathrm{BrS}$. There were no predictors of a positive ajmaline provocation test on multivariable analysis. One patient who tested negative at 12 years of age, subsequently tested positive at 15 years of age.

Conclusions: Ajmaline testing appears safe and feasible in children when performed in an appropriate setting by an experienced team. Test positivity may change with age in individuals, suggesting that the test should be repeated in the late teenage years or early adulthood.

\section{INTRODUCTION}

The Brugada syndrome $(\mathrm{BrS})$ is an inherited arrhythmia syndrome that is a cause of sudden cardiac death (SCD) in adults and children. ${ }^{1-3}$ It is characterised by a stereotypical ECG pattern of cove-shaped ST segment elevation in the anterior chest leads, ${ }^{1}{ }^{4-6}$ although this pattern is not always apparent, even in individuals with confirmed BrS. The prevalence of this pattern increases with age, suggesting" that there are children with the syndrome who will go on to develop a diagnostic ECG pattern. The diagnostic pattern fluctuates ${ }^{7}$ and can also be unmasked by other factors such as fever and medications. ${ }^{9}$

The use of class Ia antiarrhythmic agents such as flecainide and ajmaline to unmask occult $\mathrm{BrS}$ in the adult population has been extensively reported..$^{9-12}$ Ajmaline has been selected as the agent of choice due to its short half-life, established safety profile in adults and higher diagnostic yield compared with flecainide. ${ }^{13}$ However, little data exist on its safety and efficacy in children. ${ }^{14}$ This study aims to add further safety and feasibility data on the diagnostic ajmaline provocation test in children in a specialist paediatric inherited cardiovascular diseases centre.

\section{METHODS}

\section{Ajmaline test protocol}

All ajmaline tests were performed and interpreted by experienc"'"ed cardiologists with expertise in the evaluation and management of inherited cardiac conditions (ML, DA and JPK), with advanced cardiovascular resuscitation equipment to hand. All patients (or parents/guardians) gave informed consent to the test being performed, and signed an appropriate form to record this. Patients were intravenously cannulated and attached 
to continuous 12-lead ECG monitoring (using either a Mortara ECG machine via an Ultima $\mathrm{CardiO}_{2}$ workstation or a Marquette MAC 5000 ECG machine). Baseline ECGs were taken after a period of rest. The ECGs were analysed at $1 \mathrm{~min}$ intervals throughout the duration of the test. Ajmaline was given intravenously as a phased infusion over $5 \mathrm{~min}$. A dose of $1 \mathrm{mg} / \mathrm{kg}$ up to a maximum of $50 \mathrm{mg}$ was used. The infusion was discontinued if the ECGs became diagnostic or the QRS duration increased by $150 \%$ or more. ${ }^{11}$ ECGs were monitored until the PR interval and QRS durations had normalised. A test was considered positive if there was J point elevation of $\geq 2 \mathrm{mms}$ with coved ST elevation in more than one right precordial lead. ${ }^{6}{ }^{11}$ From 2011 onwards, all patients undergoing ajmaline provocation testing $(n=24(24 \%))$ routinely had ECGs recorded in leads V1 and V2 in the high parasternal position in addition to the standard lead placement.

Individuals with a positive ajmaline provocation test received advice in relation to fever management and avoidance of drugs with the potential to precipitate ventricular arrhythmias in this population, and were advised to undergo an ECG during febrile episodes. ${ }^{15}$ All individuals were followed at 6-12 monthly intervals by the same team at our institution with a resting and/or ambulatory ECG.

Structural heart disease was excluded in all patients prior to testing using non-invasive imaging (echocardiography and/or cardiac MRI) as has previously been described. ${ }^{16}$ Genetic testing for mutations in the SCN5A gene was not routinely performed.

\section{ECG analysis}

Available ECGs were further analysed retrospectively by a single investigator (MRM) at pretest baseline and maximal effect of ajmaline, defined by maximal prolongation of ECG parameters. The PR, QRS, QT and RR intervals were measured using vernier callipers (accurate to $0.02 \mathrm{~mm}$ ) and averaged across three heart beats in leads II and V5 $(10,14)$. The QTc was calculated using Bazett's formula. ${ }^{17}$ Patients were classified as having partial right bundle branch block (RBBB) if there was an $\mathrm{Rsr}^{\mathrm{I}}$ pattern in $\mathrm{V} 1$ or V2 without prolongation of the QRS beyond $120 \mathrm{~ms}$. No patients had a complete RBBB.

\section{Statistical analysis}

All statistical analyses were performed using R V.12.2.1 (R Foundation for Statistical Computing, Austria). ${ }^{18}$ Normally distributed, continuous data are presented as mean $( \pm \mathrm{SD})$ with categorical data presented as $\mathrm{n}(\%)$. Two sample two-tailed Welch t tests were used to compare means between groups, with paired two-tailed Welch $\mathrm{t}$ tests used to test before and after ajmaline provocation testing in intragroup testing. $\chi^{2}$ Tests were used to compare categorical data. To further explore the difference between negative and positive ajmaline test groups, multiple logistic modelling was performed. Positivity of the ajmaline test was used as the outcome measure, with combinations of baseline HR, PR, QRS and QTc with age and gender used as potential predictors. A value of $\mathrm{p}<0.05$ was considered significant in all cases.

\section{RESULTS}

Ninety-five consecutive patients aged 18 years or below (12.55 years, SD 3.34; range 5-18 years and 4 months, 1 outlier at 1 year 10 months) underwent ajmaline provocation testing in the Inherited Cardiovascular Diseases Unit at Great Ormond Street Hospital from the 1 September 2004 to 31 July 2012. Forty-one patients were female $(43 \%)$. Three patients were retested in the department at an average of 5 years after the initial test, due to the possibility of age-related penetrance. Baseline characteristics of these patients are presented in table 1 . No patients had evidence of a spontaneous or feverinduced type I Brugada ECG during follow-up.

The indication for ajmaline challenge was a family history of $\mathrm{BrS}$ in 46 patients $(48 \%)$, family history of unexplained SCD (without a confirmed family history of $\mathrm{BrS}$ ) in $39(41 \%)$, symptoms with suspicious ECG abnormalities in 9 (9\%; syncope, $n=5$; chest pain, $n=1$; palpitations, $n=3$ ) and development of bradycardia with abnormal ECG during general anaesthesia with propofol in 1 patient. An infant was admitted following an apparent life-threatening event after her brother had died in confirmed ventricular fibrillation (VF). This child underwent an ajmaline test in the catheter lab at the age of 1 year and 10 months while having a loop recorder device implanted.

One patient had evidence of sinus node dysfunction and atrial flutter at presentation; all other patients were in sinus rhythm, with no evidence of conduction disease or atrial arrhythmia.

\section{Results of ajmaline provocation testing}

A diagnostic type I response was reported in 19 individuals (20\%). Thirteen of the 46 patients (29\%) who underwent ajmaline provocation testing for a family history of $\mathrm{BrS}$ tested positive, compared with 5 of the 39 (13\%) patients tested for a family history of SCD. The remaining positive result was in an individual presenting with syncope and a suspicious ECG. Of the 13 patients with a positive ajmaline test referred because of a family history of BrS, there was a history of SCD in a second degree relative in one family only.

Of the positive ajmaline tests, 12 patients' ECGs $(63 \%)$ showed a diagnostic pattern within 4 min of the start of the infusion (shortest time $55 \mathrm{~s}$ ), and a further 4 became positive after $5 \mathrm{~min}$. No patients developed a diagnostic pattern after 6 min of infusion of ajmaline.

\section{ECG characteristics following ajmaline infusion}

ECG characteristics are shown in table 2. PR, QRS and QTc all changed significantly following the administration of ajmaline with patients with a positive test result experiencing a larger prolongation of QTc $(\mathrm{p}<0.01)$. 
Table 1 Baseline characteristics of 95 children undergoing ajmaline provocation testing

\begin{tabular}{|c|c|c|c|c|}
\hline & BrS-positive result & BrS-negative result & $\begin{array}{l}\text { p Value comparing } \\
\text { positive vs negative }\end{array}$ & Total \\
\hline $\mathrm{N}$ & 19 & 76 & & 95 \\
\hline Mean age (SD) & $11.87(3.1)$ & $12.8(3.37)$ & 0.266 & $12.55(3.34)$ \\
\hline Age range & $7.4-16.4$ & $1.9-18.4$ & & $1.9-18.4$ \\
\hline Indications & 13 & 33 & $0.08^{*}$ & 45 (48\%) \\
\hline \multicolumn{5}{|l|}{ Family history of BrS (n) } \\
\hline Family history of SCD (n) & 5 & 34 & & $39(41 \%)$ \\
\hline Symptomatic $(n)$ & 1 & 8 & & $9(9 \%)$ \\
\hline Partial RBBB on ECG† & $7 / 16(44 \%)$ & $23 / 71(32 \%)$ & 0.3732 & $30 / 87(34 \%)$ \\
\hline
\end{tabular}

Although the report from the ajmaline challenge was available in all patients' notes, the hard copy of the ECG tracings was unavailable for further retrospective review in 12 patients; in 8 patients the printout was not present, and in a further 4 patients the ECGs available were of insufficient quality to measure accurately.

On multivariable analysis, prolongation of the PR interval was associated with an increased probability of ajmaline test positivity in one model, but overall there were no clinically significant predictors of a positive ajmaline test identified, most likely due to the sample size being too small to detect significant changes (see supplementary material).

\section{Safety of ajmaline provocation testing in children}

No adverse events were detected in the 98 ajmaline tests performed. In particular, there were no documented arrhythmias or cardiovascular symptoms related to the ajmaline test. The infusion was discontinued before achieving the target dose in the 12 patients that showed ECG changes before $4 \mathrm{~min}$.

\section{Clinical outcome following ajmaline provocation testing}

The mean follow-up time from the time of ajmaline testing was 3.66 years, with a total follow-up time of 341 patient-years. During this time, no patient died, experienced a documented arrhythmia, non-vasovagal syncope or other cardiac symptoms. In total, 5 individuals $(26 \%)$ with a positive ajmaline test underwent diagnostic electrophysiological studies (EPS) with ventricular tachycardia (VT) stimulation, all prior to 2006; no ventricular arrhythmias were induced. From 2006 onwards, diagnostic EPS VT stimulation studies have not been routinely performed in children with a positive ajmaline provocation test in our institution, according to published guidelines. $^{19}$ One further patient underwent an EPS VT stimulation study during an episode of atrial flutter.

One patient experienced a change in their ajmaline test result. She was referred to the service following the SCD of her father in his fifth decade. Initial ajmaline testing was carried out at 12 years of age and was non-diagnostic, showing a borderline type 2 response (figure 1A). The patient was retested at 15 years of age and showed a

Table 2 ECG characteristics by result of ajmaline test

\begin{tabular}{|c|c|c|c|c|}
\hline & Before mean (SD) & After mean (SD) & Difference mean (SD) & p Value \\
\hline \multicolumn{5}{|c|}{ (A) ECG parameters before and after the ajmaline challenge } \\
\hline Heart rate (bpm) & $87(16)$ & $95(14)$ & $7(2)$ & $<0.001$ \\
\hline PR interval (ms) & $143(24)$ & $196(34)$ & $53(5)$ & $<0.001$ \\
\hline QRS (ms) & $85(14)$ & $119(18)$ & $34(4)$ & $<0.001$ \\
\hline QTc (ms) & $412(21)$ & $461(27)$ & $49(6)$ & $<0.001$ \\
\hline \multicolumn{5}{|c|}{ (B) In patients with positive test results } \\
\hline Heart rate (bpm) & $83(10)$ & $91(10)$ & $8(4)$ & 0.0015 \\
\hline PR interval (ms) & $154(25)$ & $212(26)^{*}$ & $58(12)$ & $<0.001$ \\
\hline QRS (ms) & $89(15)$ & $125(15)$ & $37(7)$ & $<0.001$ \\
\hline QTc (ms) & $411(27)$ & $477(34)^{*}$ & $66(18)^{*}$ & $<0.001$ \\
\hline \multicolumn{5}{|c|}{ (C) In patients with negative test results } \\
\hline Heart rate (bpm) & $88(17)$ & $95(15)$ & $7(3)$ & $<0.001$ \\
\hline PR interval (ms) & $141(23)$ & $192(35)^{*}$ & $52(6)$ & $<0.001$ \\
\hline QRS (ms) & $84(14)$ & $117(19)$ & $33(4)$ & $<0.001$ \\
\hline QTc (ms) & $412(24)$ & $457(24)^{*}$ & $46(5)^{\star}$ & $<0.001$ \\
\hline
\end{tabular}



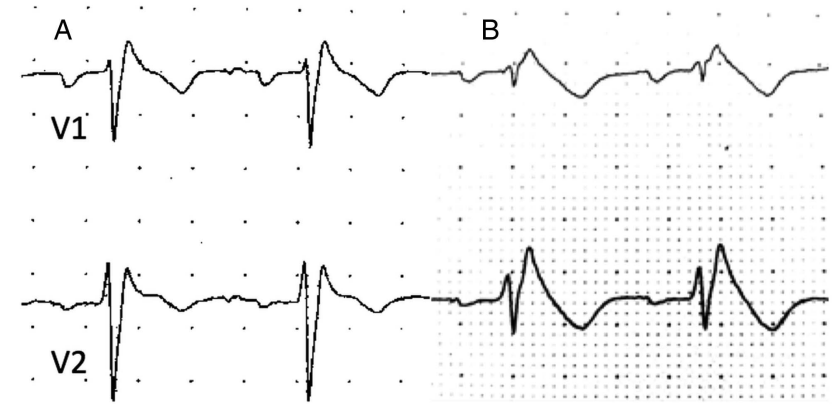

Figure 1 Variation in ajmaline response in a female patient. (A) Ajmaline test performed at 12 years of age, showing no $\mathrm{J}$-point elevation in the anterior precordial leads. (B) Ajmaline test performed at 15 years of age, showing a type I Brugada syndrome ECG pattern in leads V1 and V2.

diagnostic type 1 response (figure 1B). She remains asymptomatic, with no evidence of a spontaneous type I Brugada ECG, but subsequently other adult members of the family have had positive ajmaline tests.

Another patient presented with syncope secondary to documented atrial flutter. There was a strong family history of BrS including in his father, who on genotyping was positive for the c.4346A $>\mathrm{G}$ ( p.Tyr1449Cys) in exon 25 of the SCN5A gene. This is a previously reported mutation $^{20}$ absent in control DNA and predicted to be pathogenic. On referral to the department, aged 14, his baseline ECG showed sinus node disease and ajmaline provocation testing was positive (figure 2). On genotyping the patient was also positive for the mutation, which segregated for disease in this family. He underwent two flutter ablation procedures; VT stimulation study was negative.

\section{DISCUSSION}

This study represents the largest series yet published of ajmaline provocation testing in a paediatric population. It shows that ajmaline provocation testing is feasible and can be used safely in the investigation of $\mathrm{BrS}$ in children, when performed in the setting of a specialist paediatric inherited cardiac conditions centre. The lack of arrhythmic episodes is in keeping with the literature available in both children ${ }^{14}$ and adults. ${ }^{11}{ }^{21}$ We also describe the first reported incidence of the ajmaline provocation test result changing for an individual.

\section{Safety considerations}

Ajmaline has been in use as an antiarrhythmic medication for over five decades ${ }^{22}$ and its safety profile in adults is well known. Recently, ajmaline has been used as a diagnostic tool to unmask latent $\mathrm{BrS} .{ }^{11}$ An early case report showed a death secondary to ventricular tachyarrhythmia. ${ }^{23}$ Rolf et al ${ }^{11}$ set out to develop a test protocol to minimise risk of arrhythmias, with a prevalence of $1.3 \%$ for VT. In a subsequent larger, retrospective study, Veltmann $e t a l^{12}$ showed a much lower rate of VT/VF at $0.15 \%$, attributed to the adoption of the new protocol. Data in the paediatric population are scarce, but a recent study involving 28 children demonstrated no ventricular arrhythmias ${ }^{14}$; the present study confirms the low risk of ajmaline provocation testing in children, and highlights the importance of performing the study in an appropriate setting by an experienced team and with access to advanced life support equipment and medication $^{11}$ and with the capability to provide appropriate interpretation and management expertise.
Figure 2 ECG features in a teenager with a pathogenic SCN5A mutation. (A) Resting ECG showing sinus node dysfunction; (B) ECG showing atrial flutter; $(C)$ ajmaline provocation test with $\mathrm{V} 1$ and $\mathrm{V} 2$ leads in the standard position; (D) ajmaline provocation test with $\mathrm{V} 1$ and V2 leads in the high-parasternal position, showing diagnostic J-point elevation.
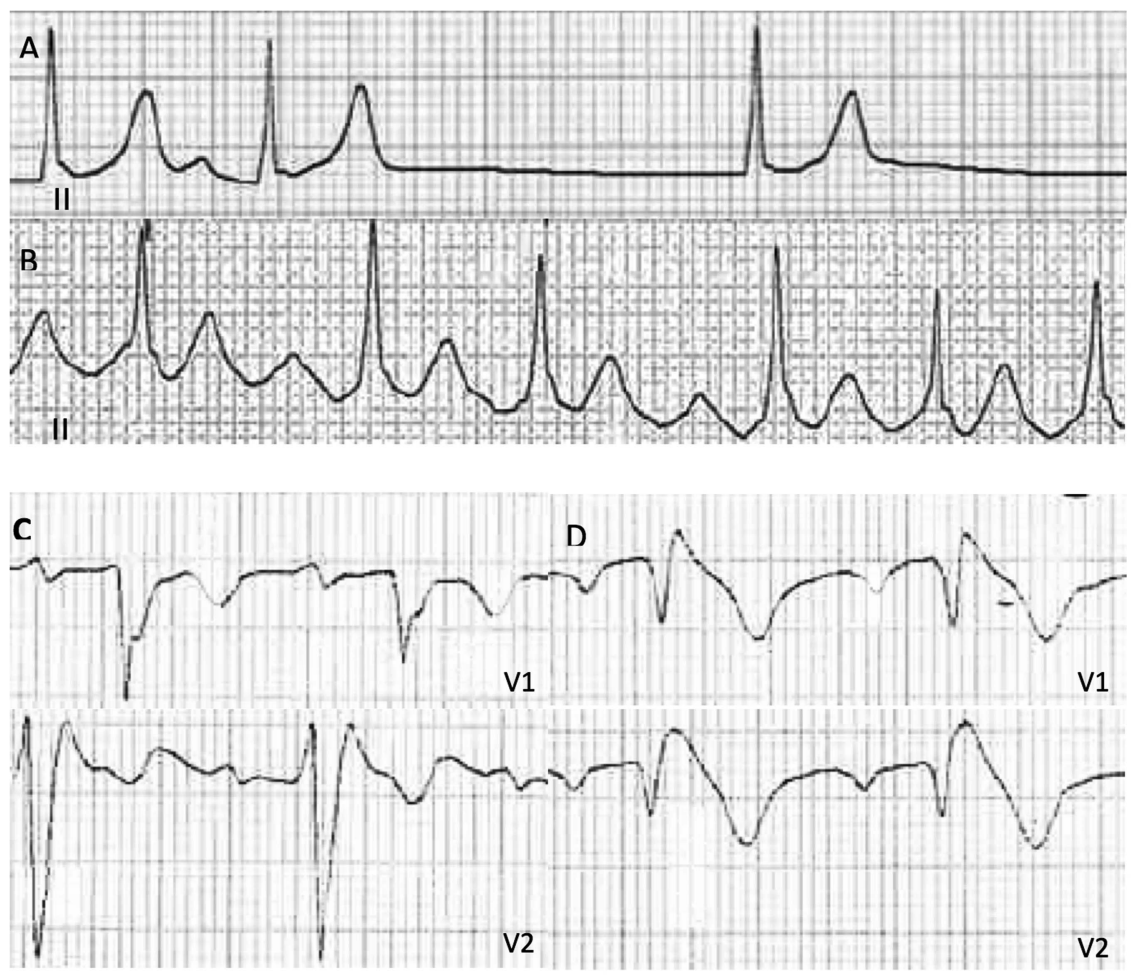


\section{Results of ajmaline testing}

The prevalence of a diagnostic BrS ECG pattern in the present study is compatible to previous reports in adults undergoing ajmaline provocation testing ${ }^{11}{ }^{12}$ but substantially lower than the only other report of ajmaline provocation testing in children $(54 \%$ of 28 paediatric patients). ${ }^{14}$ This apparent discrepancy likely represents a difference in the population studied, with the majority of patients in the study by Sorgente $e t a l^{14}$ undergoing ajmaline testing following the diagnosis of $\mathrm{BrS}$ in a first-degree relative. In the present study, nearly one third of patients undergoing ajmaline testing for a family history of $\mathrm{BrS}$ in a first-degree relative had a diagnostic type I ECG pattern, consistent with the autosomal dominant inheritance pattern with variable penetrance of this condition.

Although we found no clinically important predictors of a positive ajmaline provocation challenge, there was a trend towards a difference in PR duration, with greater prolongation of the $\mathrm{PR}$ interval in individuals with a positive ajmaline test. SCN5A mutations have been found in association with sick sinus syndrome, cardiac conduction disease and atrial fibrillation ${ }^{15}$; the findings in this study are in keeping with the presence of conduction disease in the pathophysiology of $\mathrm{BrS}$.

A novel finding in this study is the apparent change from non-diagnostic to diagnostic response to ajmaline in a single individual. While the presence of false negative ajmaline tests is well-recognised, ${ }^{10}$ this finding raises the possibility of an age-related penetrance to the ajmaline provocation test, and supports the need to retest in late adolescence or early adulthood in individuals with a negative ajmaline test in earlier childhood. The presence of age-related penetrance may be related to hormonal effects on ion channel expression or may represent the progression of myocardial fibrosis with time. Larger prospective studies are required to further evaluate the prevalence of age-related changes and efficacy of this approach.

\section{Long-term outcomes of BrS}

Although this study did not set out to investigate the clinical outcome of $\mathrm{BrS}$ in children, the results suggest that it generally has a benign prognosis in childhood, similar to previous reports. ${ }^{24}$ Nevertheless, BrS can cause SCD in children ${ }^{325} 26$ and further prospective data are needed to improve risk stratification in this population.

In our population there were no changes in treatment or interventions performed in light of a positive ajmaline test. This introduces the question as to whether screening for $\mathrm{BrS}$ should be offered to asymptomatic children at all. On the one hand, the finding of a positive ajmaline test may allow appropriate management, for example by implantation of an implantable cardiac defibrillator in cases thought to be at high risk (eg, those presenting with syncope), and there are emerging data on the possible efficacy of quinidine in this population. ${ }^{27-30}$ Furthermore, as $\mathrm{BrS}$ is inherited as an autosomal dominant trait, confirmation of the diagnosis in a child of an individual known to have $\mathrm{BrS}$ can remove the uncertainty of a situation in which there is a priori a $50 \%$ chance of inheriting the condition. Conversely, a negative ajmaline test can provide peace of mind to the family.

On the other hand, a positive test may cause significant psychological distress without changing clinical management, and a negative test may not completely eliminate uncertainty, due to the possibility of age-related changes. Further research is required to determine the prognostic value of ajmaline testing in childhood and to allow paediatric-specific guidelines to be developed. In the mean time, each case and family should be evaluated on an individual basis with careful consideration of the potential benefits and disadvantages of screening for $\mathrm{BrS}$ in a child, and there is an argument for suggesting that ajmaline testing could be delayed until adulthood in the absence of symptoms. In this context, it is essential that children from families with $\mathrm{BrS}$ and sudden arrhythmic death syndrome are evaluated and managed in highly specialised centres with specific expertise in the assessment and treatment of children with inherited cardiac conditions, and with access to specialist medical, nursing, counselling and psychological support to allow appropriate interpretation and subsequent management of investigations such as the ajmaline test. ${ }^{16}$

\section{Limitations}

This study is limited by its retrospective nature, resulting in not all ECGs being available for retrospective analysis. However, electronic data records ensured that there were accurate data on the results of the test in all cases, and the results of the ajmaline tests in particular were reported at the time of the test. A further limitation caused by the retrospective design is that these data were collected over an 8-year period, during which clinical practice and knowledge have altered in our institution and throughout the inherited cardiovascular diseases community. In particular, recording of high leads has only routinely been carried out in our patients in the past 2 years, which may affect the sensitivity of the test. In addition, the use of a maximum dose of $50 \mathrm{mg}$ may also result in reduced diagnostic value of this test in older teenagers weighing more than $50 \mathrm{~kg}$. Only one investigator (MRM) formally analysed the ECGs, meaning it was not possible to calculate interobserver variability. However, it is worth noting that all the ECGs were also analysed at the time of the test by an experienced consultant cardiologist (ML, DA and JPK), and no discrepancies in clinical interpretation were identified. In addition, the relatively small numbers of positive tests did not allow meaningful analysis of predictors. The study cohort has not been systematically genotyped, therefore not allowing an assessment of specificity and sensitivity of the ajmaline provocation test to be made. However, as the diagnostic yield of genetic testing for $\mathrm{BrS}$ is approximately $20 \%^{31}$ it is likely that most of the individuals in this study would not have been found to harbour mutations in known BrS-causing genes. 
Furthermore, previous studies have demonstrated a high sensitivity of the ajmaline test ${ }^{13}$ although, in the absence of a gold standard for the diagnosis of $\mathrm{BrS}$, calculations of accuracy must be interpreted with extreme caution.

\section{CONCLUSIONS}

This study shows that ajmaline provocation testing appears to be safe and feasible in the paediatric population when performed in an appropriate setting by an experienced team. A diagnostic (type I) response is more common in patients with a family history of $\mathrm{BrS}$ in a first-degree relative, and there may be an age-related penetrance to the test. Further studies are required to assess the prognostic value of ajmaline provocation testing in childhood.

\section{Author affiliations}

${ }^{1}$ Inherited Cardiovascular Diseases Unit, Department of Cardiology, Great Ormond Street Hospital for Children, London, UK

${ }^{2}$ Department of Cardiology, Boston Children's Hospital, Boston, Massachusetts, USA

${ }^{3}$ Institute of Cardiovascular Science, University College London, London, UK

Contributors MRM collected and analysed the data and wrote the first draft of the manuscript. TGD, MB, JM and ML collected and analysed the data and approved the final version of the manuscript. SM-R and RB collected the data and approved the final version of the manuscript. DA collected and analysed the data, revised the draft and approved the final version of the manuscript. JPK designed the study, collected the data, analysed the data, revised the manuscript and approved the final version for publication. JPK is the guarantor.

Funding This research received no specific grant from any funding agency in the public, commercial or not-for-profit sectors.

Competing interests None.

Provenance and peer review Not commissioned; externally peer reviewed.

Data sharing statement No additional data are available.

Open Access This is an Open Access article distributed in accordance with the Creative Commons Attribution Non Commercial (CC BY-NC 3.0) license, which permits others to distribute, remix, adapt, build upon this work noncommercially, and license their derivative works on different terms, provided the original work is properly cited and the use is non-commercial. See: http:// creativecommons.org/licenses/by-nc/3.0/

\section{REFERENCES}

1. Brugada P, Brugada J. Right bundle branch block, persistent ST segment elevation and sudden cardiac death: a distinct clinical and electrocardiographic syndrome. A multicenter report. J Am Coll Cardiol 1992;20:1391-6.

2. Chen $Q$, Kirsch GE, Zhang D, et al. Genetic basis and molecular mechanism for idiopathic ventricular fibrillation. Nature 1998;392:293-6.

3. Priori SG, Napolitano C, Giordano U, et al. Brugada syndrome and sudden cardiac death in children. Lancet 2000;355:808-9.

4. Atarashi H, Ogawa S. New ECG criteria for high-risk Brugada syndrome. Circ J 2003;67:8-10.

5. Bayes de Luna A, Brugada J, Baranchuk A, et al. Current electrocardiographic criteria for diagnosis of Brugada pattern: a consensus report. J Electrocardiol 2012:45:433-42.

6. Wilde AA, Antzelevitch C, Borggrefe M, et al. Proposed diagnostic criteria for the Brugada syndrome: consensus report. Circulation 2002;106:2514-19.
7. Santos LF, Correia E, Rodrigues B, et al. Spontaneous fluctuations between diagnostic and nondiagnostic ECGs in Brugada syndrome screening: Portuguese family with Brugada syndrome. Ann Noninvasive Electrocardiol 2010;15:337-43.

8. Veltmann C, Schimpf R, Echternach C, et al. A prospective study on spontaneous fluctuations between diagnostic and non-diagnostic ECGs in Brugada syndrome: implications for correct phenotyping and risk stratification. Eur Heart J 2006;27:2544-52.

9. Arnalsteen-Dassonvalle E, Hermida JS, Kubala M, et al. Ajmaline challenge for the diagnosis of Brugada syndrome: which protocol? Arch Cardiovasc Dis 2010;103:570-8.

10. Hong K, Brugada J, Oliva A, et al. Value of electrocardiographic parameters and ajmaline test in the diagnosis of Brugada syndrome caused by SCN5A mutations. Circulation 2004;110:3023-7.

11. Rolf $\mathrm{S}$, Bruns $\mathrm{HJ}$, Wichter $\mathrm{T}$, et al. The ajmaline challenge in Brugada syndrome: diagnostic impact, safety, and recommended protocol. Eur Heart J 2003;24:1104-12.

12. Veltmann C, Wolpert C, Sacher F, et al. Response to intravenous ajmaline: a retrospective analysis of 677 ajmaline challenges. Europace 2009;11:1345-52.

13. Wolpert C, Echternach C, Veltmann C, et al. Intravenous drug challenge using flecainide and ajmaline in patients with Brugada syndrome. Heart Rhythm 2005;2:254-60.

14. Sorgente A, Sarkozy A, De Asmundis C, et al. Ajmaline challenge in young individuals with suspected Brugada syndrome. Pacing Clin Electrophysiol 2011;34:736-41.

15. Li A, Behr ER. Brugada syndrome: an update. Future Cardiol 2013;9:253-71.

16. Nunn LM, Lambiase PD. Genetics and cardiovascular diseasecauses and prevention of unexpected sudden adult death: the role of the SADS clinic. Heart 2011;97:1122-7.

17. Bazett HC. An analysis of the time-relations of electrocardiograms. Heart 1920;7:353-70.

18. The R Development Core Team. $R$ : A language and environment for statistical computing. 2010.

19. Paul M, Gerss J, Schulze-Bahr E, et al. Role of programmed ventricular stimulation in patients with Brugada syndrome: a meta-analysis of worldwide published data. Eur Heart $J$ 2007:28:2126-33.

20. Kapplinger JD, Tester DJ, Alders $M$, et al. An international compendium of mutations in the SCN5A-encoded cardiac sodium channel in patients referred for Brugada syndrome genetic testing. Heart Rhythm 2010;7:33-46.

21. Fish JM, Antzelevitch C. Role of sodium and calcium channel block in unmasking the Brugada syndrome. Heart Rhythm 2004;1:210-17.

22. Arora RB, Madan BR. Antiarrhythmics. Vi. Ajmaline and serpentine in experimental cardiac arrhythmias. $J$ Pharmacol Exp Ther 1956;117:62-7.

23. Wellens HJ, Bar FW, Vanagt EJ. Death after ajmaline administration. Am J Cardiol 1980;45:905

24. Viskin S. Brugada syndrome in children: don't ask, don't tell? Circulation 2007:115:1970-2.

25. Doetzer AD, Sotomaior VS, Bubna MH, et al. What can be done when asymptomatic patients discover they have Brugada syndrome? A case report of Brugada syndrome. Int J Cardiol 2011;150:e96-7.

26. Mivelaz Y, Di Bernardo S, Pruvot E, et al. Brugada syndrome in childhood: a potential fatal arrhythmia not always recognised by paediatricians. A case report and review of the literature. Eur $J$ Pediatr 2006;165:507-11

27. Belhassen $B$. Is quinidine the ideal drug for Brugada syndrome? Heart Rhythm 2012;9:2001-2.

28. Marquez MF, Bonny A, Hernandez-Castillo E, et al. Long-term efficacy of low doses of quinidine on malignant arrhythmias in Brugada syndrome with an implantable cardioverter-defibrillator: a case series and literature review. Heart Rhythm 2012;9:1995-2000.

29. Minoura Y, Panama BK, Nesterenko VV, et al. Effect of Wenxin Keli and quinidine to suppress arrhythmogenesis in an experimental model of Brugada syndrome. Heart Rhythm 2013;10:1054-62.

30. Viskin S, Wilde AA, Guevara-Valdivia ME, et al. Quinidine, a life-saving medication for Brugada syndrome, is inaccessible in many countries. J Am Coll Cardiol 2013:61:2383-7.

31. Crotti L, Marcou CA, Tester DJ, et al. Spectrum and prevalence of mutations involving BrS1- through BrS12-susceptibility genes in a cohort of unrelated patients referred for Brugada syndrome genetic testing: implications for genetic testing. J Am Coll Cardiol 2012:60:1410-18. 